\title{
Renal Remission Status and Longterm Renal Survival in Patients with Lupus Nephritis: A Retrospective Cohort Analysis
}

\author{
Julie E. Davidson, Qinggong Fu, Beulah Ji, Sapna Rao, David Roth, Laurence S. Magder, \\ and Michelle Petri
}

ABSTRACT. Objective. This observational study was a retrospective analysis of prospectively collected Hopkins Lupus Cohort data to compare longterm renal survival in patients with lupus nephritis (LN) who achieved complete (CR), partial (PR), or no remission following standard-of-care LN induction therapy.

Methods. Eligible patients with biopsy-proven LN (revised American College of Rheumatology or Systemic Lupus Collaborating Clinics criteria) were identified and categorized into ordinal (CR, PR, or no remission) or binary (response or no response) renal remission categories at 24 months postdiagnosis [modified Aspreva Lupus Management Study (mALMS) and modified Belimumab International Lupus Nephritis Study (mBLISS-LN) criteria]. The primary endpoint was longterm renal survival [without endstage renal disease (ESRD) or death].

Results. In total, 176 patients met the inclusion criteria. At Month 24 postbiopsy, more patients met mALMS remission criteria $(C R=59.1 \%, P R=30.1 \%)$ than mBLISS-LN criteria $(C R=40.9 \%$, $\mathrm{PR}=16.5 \%$ ). During subsequent followup, 18 patients developed ESRD or died. Kaplan-Meier plots suggested patients with no remission at Month 24 were more likely than those with PR or CR to develop the outcome using either mALMS $(p=0.0038)$ and mBLISS-LN $(p=0.0097)$ criteria for remission. Based on Cox regression models adjusted for key confounders, those in CR according to the mBLISS-LN (HR $0.254,95 \%$ CI $0.082-0.787 ; \mathrm{p}=0.0176)$ and mALMS criteria (HR 0.228, $95 \%$ CI $0.063-0.828 ; \mathrm{p}=0.0246$ ) were significantly less likely to experience ESRD/mortality than those not in remission.

Conclusion. Renal remission status at 24 months following LN diagnosis is a significant predictor of longterm renal survival, and a clinically relevant endpoint. (First Release March 1 2018; J Rheumatol 2018;45:671-7; doi:10.3899/jrheum.161554)

Key Indexing Terms: ENDSTAGE RENAL DISEASE MORTALITY

\section{COHORT STUDIES REMISSION INDUCTION}

\section{LUPUS NEPHRITIS SURVIVAL}

\begin{abstract}
Although there have been improvements in both the diagnosis and treatment of patients with systemic lupus erythematosus (SLE) over recent decades, lupus nephritis $(\mathrm{LN})$ remains an indicator of poor prognosis ${ }^{1,2,3}$. Active $\mathrm{LN}$ progresses to endstage renal disease (ESRD) in about $10-30 \%$ of patients ${ }^{4}$. Patients with LN who develop ESRD have a much higher mortality rate than those who do not
\end{abstract}

develop ESRD ${ }^{5,6}$, with renal injury considered the most important predictor of mortality in patients with $\mathrm{SLE}^{4}$.

Clinical trials in $\mathrm{LN}$ have been designed to demonstrate induction of renal remission (as a measure of response to therapy) based on various laboratory measures of renal function and activity. These measures have been used to create clinical endpoints based on the degree of renal
From the Real World Evidence and Clinical Development, GlaxoSmithKline R\&D, Stockley Park, Uxbridge, UK; GlaxoSmithKline $R \& D$, Upper Providence, Pennsylvania, and Research Triangle Park, North Carolina; the Department of Epidemiology and Public Health, University of Maryland; Division of Rheumatology, Johns Hopkins University School of Medicine, Baltimore, Maryland, USA.

This study was funded by GlaxoSmithKline (GSK). The Hopkins Lupus Cohort was funded by the US National Institutes of Health (AR 43727 and AR 69572). JED was a shareholder of GSK and an employee of GSK at the time of the study, and is a current employee of Eli Lilly and Company Ltd. $Q F$ is a shareholder and an employee of GSK. BJ is a shareholder and an employee of GSK. SR is an employee of GSK. DR is a shareholder and an employee of GSK.
J.E. Davidson*, PhD, MPH, Real World Evidence, GlaxoSmithKline $R \& D ; Q . F u, P h D$, Real World Evidence, GlaxoSmithKline R\&D; B. Ji, $M D$, Clinical Development, GlaxoSmithKline R\&D; S. Rao, MS, Real World Evidence, GlaxoSmithKline R\&D; D. Roth, MD, Clinical Development, GlaxoSmithKline R\&D; L.S. Magder, PhD, Department of Epidemiology and Public Health, University of Maryland; M. Petri, MD, Division of Rheumatology, Johns Hopkins University School of Medicine. * Name at the time of the study.

Address correspondence to Dr. B. Ji, GSK, Stockley Park West, 1-3 Ironbridge Road, Uxbridge, Middlesex UB11 1BT, UK. E-mail:Beulah.N.Ji@gsk.com

Full Release Article. For details see Reprints/Permissions at jrheum.org Accepted for publication November 28, 2017.

Personal non-commercial use only. The Journal of Rheumatology Copyright (c) 2018. All rights reserved. 
response to treatment at a specific timepoint, including an ordinal endpoint [no remission, partial remission (PR), and complete remission $(\mathrm{CR})]^{7,8}$ or binary endpoint (response or no response $)^{8,9}$. The definitions of both CR and PR and the designated periods for which these endpoints have been assessed have varied in previous studies ${ }^{10,11,12}$; however, few studies have assessed the longterm clinical relevance of $\mathrm{PR}^{13}$.

Our present study aimed to retrospectively compare longterm renal survival in patients with $\mathrm{CR}, \mathrm{PR}$, or no remission, as defined by criteria from the Aspreva Lupus Management Study (ALMS) ${ }^{9}$ and the Belimumab International Lupus Nephritis Study (BLISS-LN), using both binary and ordinal renal remission criteria in a large clinical cohort. In our study, both these criteria were modified to exclude urinary sediments (referred to throughout as mALMS and mBLISS-LN).

The primary objectives of our study were to (1) describe renal remission following conventional therapies in $\mathrm{LN}$ at 24 months of treatment; (2) assess the proportion of patients achieving PR at 24 months and who subsequently achieved $\mathrm{CR}$ at 36 months (CR, PR, or no remission as defined by mALMS and mBLISS-LN criteria); (3) describe longterm survival by remission status at 24 months (binary and ordinal renal remission criteria); and (4) assess the association between renal remission at 24 months (binary and ordinal renal remission criteria) and longterm survival (no ESRD and/or mortality) after adjusting for potential confounders.

\section{MATERIALS AND METHODS}

Study design. This was an observational study (GSK Study WEUKBRE6068) involving retrospective analysis of the prospectively collected Hopkins Lupus Cohort study data. The study assessed longterm outcomes of LN based on the degree of renal remission following standard-of-care therapy as measured at 24 months posttreatment.
Eligible patients with LN were identified from the Hopkins Lupus Cohort records, and patients' remission status was categorized retrospectively into ordinal (CR, PR, or no remission) or binary (response or no response) renal remission categories at 24 months after $\mathrm{LN}$ diagnosis (date of positive biopsy), based on available laboratory measures recorded during standard clinical care. Patients were followed for longterm renal survival from Month 24 after LN diagnosis until the end of followup [because of outcome event, loss to followup, or the end of the study dataset (December 2013); Figure 1].

Because data were collected in a real-world observational setting, renal functional assessments were not conducted at strict timepoints or regular intervals. Therefore, a designated window of \pm 3 months was defined around the Day 0 window for measurement of baseline renal function and model covariates and the Month 24 postbiopsy date. The result most proximal to the Month 24 timepoint was selected. It was also anticipated that few patients would have renal function screening at exactly 6 months and 36 months postbiopsy. Therefore, for the Month 36 assessments, a window of \pm 3 months was applied, to allow calculation of remission status. A hierarchy was set for defining which laboratory test to use for each remission measure if more than 1 type of measure was available (Supplementary Table 1, available with the online version of this article). If more than 1 test was available during an assessment window, an algorithm based on clinical preferences was developed to select the most appropriate test.

The Hopkins Lupus Cohort study is approved on an annual basis by the Johns Hopkins University School of Medicine Institutional Review Board (Research Project Notification: NA_00039294), and informed written consent is obtained from all participants.

Patients. Eligible patients were $\geq 18$ years of age at diagnosis, had SLE according to the revised American College of Rheumatology (ACR) or the Systemic Lupus International Collaborating Clinics (SLICC) criteria ${ }^{14,15}$, plus a biopsy record of International Society of Nephrology (ISN) class III, IV, V, or mixed LN. Patients had to have made at least 1 visit to the Johns Hopkins Lupus Center in the 3-month period following biopsy and at least 1 clinic visit during the followup period (initiation of induction therapy plus $24 \mathrm{mos}$ ) to allow assessment of longterm renal outcomes. Each patient was required to have sufficient laboratory data for assessment of renal remission at 24 months. Patients with ESRD at the initiation of induction therapy were excluded.

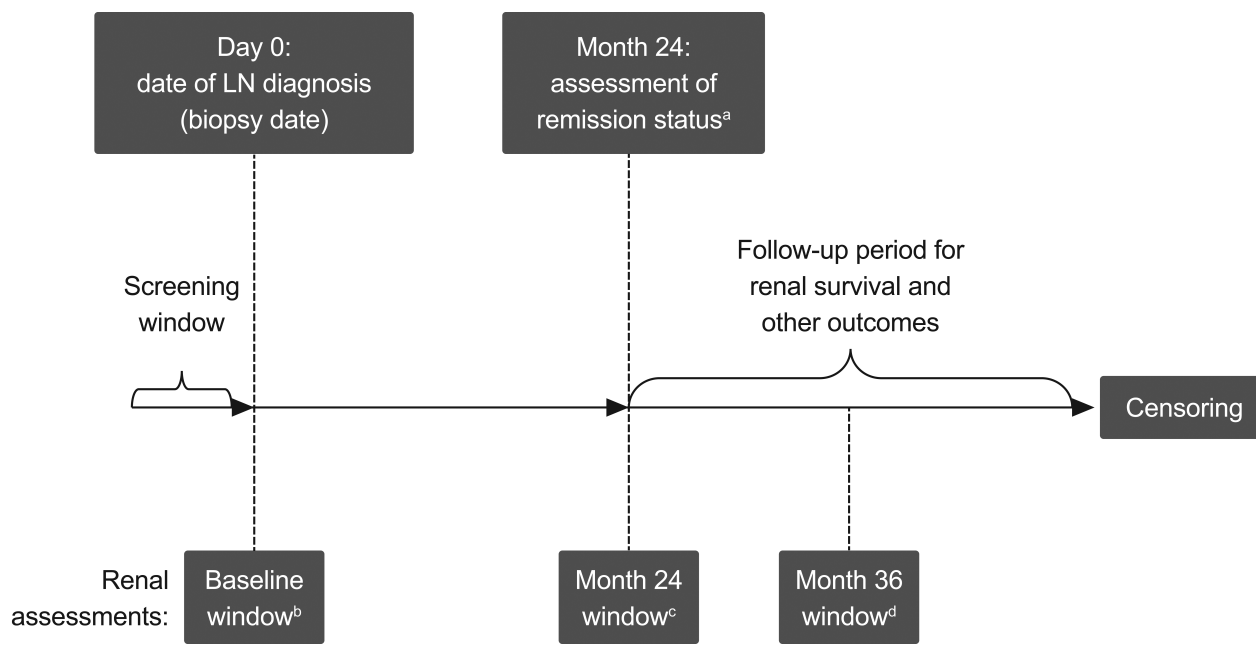

Figure 1. Study design. ${ }^{a}$ Degree of remission status achieved at Month 24 after LN biopsy data was used as the key exposure variable in this study. Renal remission status was also defined retrospectively at other study timepoints (Month 6 and Month 36). ${ }^{\mathrm{b}}$ Closest date to Day 0 (LN biopsy date) within period 3 months prior to Day 0 to 3 months after Day $0 .{ }^{\mathrm{c}}$ Closest date to Day 730 (Month 24) within a period of 3 months prior to Month 24 to 3 months after Month 24. ${ }^{\mathrm{d} C}$ losest date to Day 1095 (Month 36) within a period of 3 months prior to 3 months after Month 36. LN: lupus nephritis.

Personal non-commercial use only. The Journal of Rheumatology Copyright $\subset$ 2018. All rights reserved. 
Remission. Ordinal and binary remission categories were defined according to criteria in the ALMS (Clinicaltrials.gov identifier: NCT00377637) ${ }^{9}$ and BLISS-LN trials (Clinicaltrials.gov identifier: NCT01639339; GSK study 114054; Supplementary Table 1, available with the online version of this article). These criteria were modified (mALMS and mBLISS-LN) to exclude urinary sediment data following findings indicating that these data had low sensitivity and poor predictive value for $\mathrm{LN}$ outcomes ${ }^{16,17}$. For the current study, mALMS and mBLISS-LN criteria were applied at 24 months.

For the ordinal remission criteria, in accordance with mALMS criteria, $\mathrm{CR}$ was defined as occurrence of a return to normal serum creatinine and urine protein $\leq 0.5 \mathrm{~g} /$ day. PR was defined as one of either a return to normal serum creatinine, or urine protein $\leq 0.5 \mathrm{~g} /$ day. As per mBLISS-LN criteria, $\mathrm{CR}$ (ordinal criteria) was defined as occurrence of estimated creatinine clearance within the normal range, and urinary protein:creatinine ratio $<0.5$. Creatinine clearance was estimated according to the Cockcroft-Gault formula $^{18}$. PR was defined as occurrence of creatinine clearance of no more than $10 \%$ below the baseline value or within normal range and $\geq 50 \%$ decrease in urinary protein:creatinine ratio to $<1.0$ (if the baseline ratio were $\leq 3.0$ ) or $<3.0$ (if the baseline ratio were $>0.3$ ).

For the binary remission criteria (remission or no remission), in accordance with mALMS criteria, remission was defined as a decrease in urine protein:creatinine ratio to $<3$ in patients with baseline nephritic range proteinuria ( $\geq 3$ urine protein:creatinine ratio), and decrease in the urine protein:creatinine ratio by $\geq 50 \%$ in patients with subnephrotic proteinuria ( $<3$ urine protein:creatinine ratio). In accordance with mBLISS-LN criteria, remission was defined as meeting either the CR or the PR ordinal criteria (no remission defined as not meeting the response criteria).

Endpoints. The primary endpoint was longterm renal survival defined as survival without ESRD or mortality. ESRD was recorded prospectively in the Hopkins Lupus cohort at each clinic visit if patients had received renal replacement therapy (dialysis or transplantation), as part of the SLICC/ACR Damage Index ${ }^{19}$. The primary predictor of interest was renal remission status (ordinal criteria: CR, PR, or no remission) according to mBLISS-LN and mALMS criteria at 24 months after the biopsy date (Figure 1). Secondary endpoints included the incidence of chronic renal insufficiency and serum creatinine over time. Chronic renal insufficiency was defined as new kidney damage or new occurrence of creatinine clearance $<60 \mathrm{ml} / \mathrm{min}$ on at least 2 consecutive measurement occasions $\geq 3$ months apart. In posthoc analyses, proteinuria status (yes/no) at Month 24 was examined to investigate any association with renal survival.

Analysis. The primary study analysis was a comparison of the rates of renal survival between patients achieving PR or CR of LN at Month 24 and those who achieved no remission. Survival analysis (Kaplan-Meier plots with log-rank test and Cox proportional hazards regression) was used to compare event rates by remission status and assess the association between remission status at 24 months and renal death (ESRD or mortality), adjusting for potential confounding variables. Because this was an observational retrospective analysis, safety data such as adverse event reporting were not collected.

Multivariate analyses. Potential confounding variables considered included ISN class (III, IV, or V lupus glomerulonephritis at date of biopsy), early remission, age at date of biopsy, sex, race/ethnicity (black vs other), and SLICC Damage Index score (Day 0 window). A model-building approach was taken to produce final models according to the following rules: each variable was added in turn; those for which the remission category HR changed by $>10 \%$ were considered confounders and entered into the model. Owing to concerns about the low number of events observed and the high number of covariates, restricted models were constructed based on assessment of confounding. The number of covariates permitted in the model was set at a maximum of 2 . Where there were $>2$ confounders identified, the 2 confounders with the greatest percent change in HR were entered.

\section{RESULTS}

Patients. Based on the retrospective review of histology records during the screening window, 520 patients in the Hopkins Lupus Cohort had a biopsy record indicating ISN class III, IV, or V LN. Of these, 176 patients with LN were eligible for inclusion in our present analysis (Table 1). The majority of these patients were female $(91.5 \% ; n=161)$, just over half of the patients were black $(53.4 \% ; n=94)$, and the distribution across the included ISN categories was similar: III $(25.0 \% ; n=44)$, IV (29.0\%; $n=51)$, V $(22.7 \% ; n=40)$, and mixed $(23.3 \% ; n=41$; Table 2$)$. Three-quarters of patients had received induction therapy in the period 6 months before or after biopsy date (Table 2).

Remission. At 24 months after biopsy, more patients met the ordinal mALMS renal remission criteria than the mBLISS-LN criteria (Table 3). However, the distribution of patients meeting the binary renal remission criteria was similar between the mALMS and mBLISS-LN criteria (Table 3).

During the subsequent followup period, 18 patients developed ESRD or died. Patients with no remission at Month 24 after biopsy (ordinal criteria) were more likely than those with PR or CR to develop ESRD according to both the mALMS ( $\mathrm{p}=0.0038$; Figure 2A) and mBLISS LN $(p=0.0097)$ criteria (Figure $2 B)$. Kaplan-Meier analyses suggested that proteinuria alone was not predictive of ESRD or mortality ( $\mathrm{p}=0.8240$; Figure $2 \mathrm{C})$.

Effects of covariates are also shown in Supplementary Table 2 (available with the online version of this article). Based on Cox regression models adjusted for key confounders, those in CR (ordinal criteria) according to both the mBLISS-LN and mALMS criteria were significantly less likely to experience ESRD/mortality than those not in remission (Supplementary Table 2). Those in PR were also less likely to experience ESRD/mortality, and while this did not reach statistical significance, the HR among those in PR defined by mBLISS was lower than that of the HR for those in CR (Supplementary Table 2).

Of 53 patients who achieved mALMS criteria PR at Month 24, 14 (26.4\%) achieved CR at Month 36; of 29 patients who achieved mBLISS-LN criteria PR at Month 24, $6(20.7 \%)$ achieved CR at Month 36.

Table 1. Attrition flow. Data are $\mathrm{n}(\%)$.

\begin{tabular}{lc}
\hline Characteristic & $\begin{array}{c}\text { Attrition Flow, } \\
\mathrm{n}=2313\end{array}$ \\
\hline Patients with SLE & $2313(100.0)$ \\
LN ISN category III/IV/V & $520(22.5)$ \\
At least 1 visit within 3 mos of biopsy date & $282(12.2)$ \\
Age $\geq 18$ yrs at biopsy date (Day 0) & $277(12.0)$ \\
Sufficient data within Day 0 window & $275(11.9)$ \\
Sufficient data within Month 24 window & $189(8.2)$ \\
At least 1 visit made in followup period (after Month 24) & $181(7.8)$ \\
No ESRD during Day 0 window & $176(7.6)$ \\
No. patients selected for this analysis & 176
\end{tabular}

ESRD: endstage renal disease; ISN: International Society of Nephrology; LN: lupus nephritis; SLE: systemic lupus erythematosus. Personal non-commercial use only. The Journal of Rheumatology Copyright (c) 2018. All rights reserved. 
Table 2. Baseline demographics and patient characteristics.

\begin{tabular}{|c|c|c|}
\hline \multirow{2}{*}{$\begin{array}{l}\text { Characteristics } \\
\text { Continuous variables }\end{array}$} & \multicolumn{2}{|c|}{ LN Patient Population, $\mathrm{n}=176$} \\
\hline & Mean (SD) & Median (min-max) \\
\hline Age at biopsy date, yrs (Day 0) & $36.1(11.81)$ & $35.0(18.00-76.00)$ \\
\hline Urinary creatinine, $\mathrm{mg} / \mathrm{dl}$ & $135.7(110.15)$ & $109.0(27.00-655.00)$ \\
\hline Urinary protein:creatinine ratio & $1.5(1.80)$ & $0.8(0-9.91)$ \\
\hline Urinary $\mathrm{RBC}(\mathrm{RBC} / \mathrm{hpf})$ & $8.6(20.07)$ & $2.5(0-100.00)$ \\
\hline \multirow[t]{4}{*}{ LN ISN class } & III & $44(25.0)$ \\
\hline & IV & $51(29.0)$ \\
\hline & V & $40(22.7)$ \\
\hline & Mixed & $41(23.3)$ \\
\hline \multirow[t]{2}{*}{ Initiated induction therapy in 6 mos before or after biopsy date } & CYC & $28(15.9)$ \\
\hline & MMF & $88(50.0)$ \\
\hline \multirow[t]{4}{*}{ Decade of biopsy } & 1989 or earlier & $2(1.1)$ \\
\hline & 1990-1999 & $36(20.5)$ \\
\hline & $2000-2009$ & $123(69.9)$ \\
\hline & 2010-2013 & $15(8.5)$ \\
\hline Sex & Female & $161(91.5)$ \\
\hline \multirow{2}{*}{ Race/ethnicity } & Black & $94(53.4)$ \\
\hline & Other & $82(46.6)$ \\
\hline \multirow[t]{5}{*}{ Urine protein dipstick* (semiquantitative) category } & 0 & $18(10.2)$ \\
\hline & 0.5 & $12(6.8)$ \\
\hline & 1 & $23(13.1)$ \\
\hline & 2 & $48(27.3)$ \\
\hline & 3 & $63(35.8)$ \\
\hline
\end{tabular}

*Dipstick results were selected for the purposes of describing the cohort at baseline, because this particular method of measuring proteinuria was complete for all patients during the baseline window. **Defined as systolic blood pressure $\geq 140$ or diastolic blood pressure $\geq 90 \mathrm{mmHg}$ on 2 or more occasions, or HTN recorded as part of the SDI at any clinic visit. *** Renal biopsy established that proteinuria was SLE-related and not diabetes-related. AZA: azathioprine; CYC: cyclophosphamide; ISN: International Society of Nephrology; LN: lupus nephritis; MI: myocardial infarction; MMF: mycophenolate mofetil; RBC/hpf: red blood cells per high power field; RTX: rituximab; SDI: SLICC Damage Index; SLICC: Systemic Lupus International Collaborating Clinics; HCQ: hydroxychloroquine; HTN: hypertension; SLE: systemic lupus erythematosus.

Chronic renal insufficiency. During the followup period, 45/176 patients developed chronic renal insufficiency. Based on Cox regression models, those in CR and PR were significantly less likely to experience chronic renal insufficiency based on the mALMS criteria (CR: HR 0.077, p < 0.0001 ; PR: HR 0.152, $p<0.0001$; Figure 3A). Similarly, those in $\mathrm{CR}$ according to the mBLISS-LN criteria (but not PR) were significantly less likely to develop chronic renal insufficiency (CR: HR 0.122, p < 0.0001; PR: HR 0.611, p = 0.1824; Figure 3B). Proteinuria at 24 months was significantly associated with chronic renal insufficiency ( $p=0.0161$; Figure 3C).

Serum creatinine. The mean (SD) serum creatinine levels remained within the normal range and stable between years 1 to 3 after start of followup for those in CR or PR at Month 24 , according to both the ordinal mALMS and mBLISS-LN criteria. For those not in remission, serum creatinine levels remained elevated, although they appeared to decrease from years 1 to 3, according to both sets of criteria (Supplementary Table 3 , available with the online version of this article).

\section{DISCUSSION}

This observational study is the first of its kind, to our knowledge, to assess the longterm outcomes of LN based on degree of renal remission achieved in a real-world setting. Regardless of definition, renal remission status at Month 24

Personal non-commercial use only. The Journal of Rheumatology Copyright (C) 2018. All rights reserved. 
Table 3. Renal remission following conventional therapy in LN at 24 months of treatment.

\begin{tabular}{lcc}
\hline Renal Remission Criteria & Type of Response & $\mathrm{n}(\%)$ \\
\hline Ordinal mALMS & CR & $104(59.1)$ \\
& PR & $53(30.1)$ \\
Ordinal mBLISS-LN & None & $19(10.8)$ \\
& CR & $72(40.9)$ \\
Binary mALMS & PR & $29(16.5)$ \\
& None & $75(42.6)$ \\
Binary mBLISS-LN & Response & $109(61.9)$ \\
& None & $64(36.4)$ \\
& Response & $101(57.4)$ \\
\hline
\end{tabular}

* Three patients lacked sufficient data to be classified by binary mALMS criteria. LN: lupus nephritis; CR: complete remission; mALMS: modified Aspreva Lupus Management Study; mBLISS-LN: modified Belimumab International Lupus Nephritis Study; PR: partial remission.

in patients with LN was a statistically significant predictor of longterm renal survival. Patients with CR 24 months after biopsy (by both mBLISS-LN and mALMS criteria) were significantly less likely to experience ESRD/mortality than patients not in remission. Further, patients with PR at 24 months after biopsy (by both mBLISS-LN and mALMS criteria) were less likely to experience ESRD/mortality than patients not in remission. Although the PR/ESRD/mortality association was not statistically significant, the result approached significance for the mBLISS-LN criteria $(\mathrm{p}=0.0599)$. These results support previous findings in a small number of patients in which PR in LN was associated with significantly improved patient and renal survival, compared with patients who had no remission ${ }^{13}$. In that previous study, renal survival at 10 years was $94 \%$ (CR) and $45 \%$ (PR) versus $19 \%$ (no remission), and the patient survival without ESRD at 10 years was $92 \%$ (CR) and $43 \%$ (PR) versus $13 \%$ (no remission) $^{13}$. The composite mBLISS-LN and $\mathrm{mALMS}$ remission scores, which included consideration of creatinine levels in addition to urinary proteinuria, did seem to perform better in predicting renal survival compared with assessment of proteinuria alone.

We also assessed the association between renal remission and chronic renal insufficiency. Patients with no remission (mALMS) appeared to have a particularly marked reduction in longterm chronic renal insufficiency-free survival, compared with those in the mALMS PR or CR groups, although this was not tested statistically. A less marked separation was observed for the mBLISS-LN criteria. Because both remission and chronic renal insufficiency are defined based on serum creatinine, this association was expected. The threshold for meeting the mALMS PR or CR criteria appeared to be lower than for meeting the mBLISS-LN criteria, perhaps explaining the poor outcomes of those remaining in the ALMS "no remission" category compared with those in the mBLISS-LN "no remission"
A

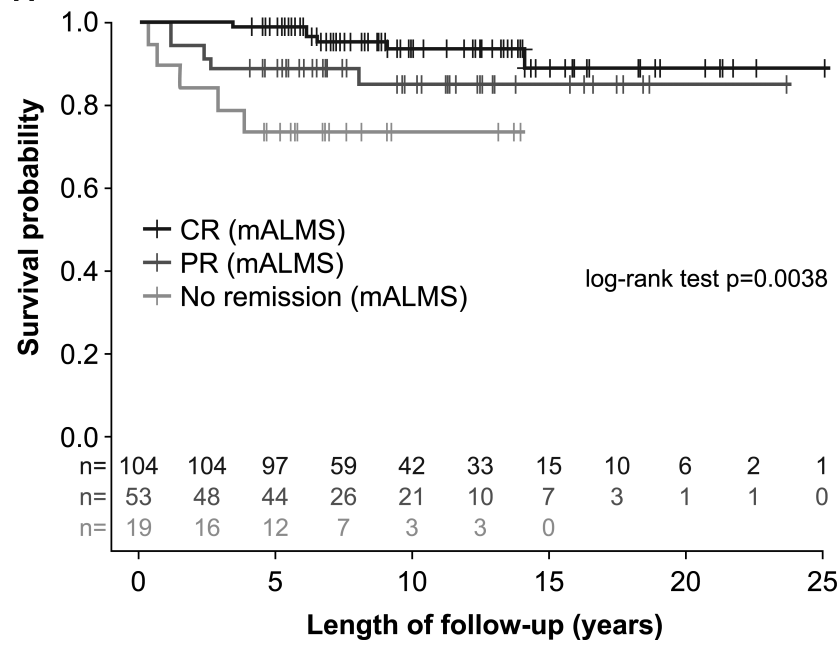

B

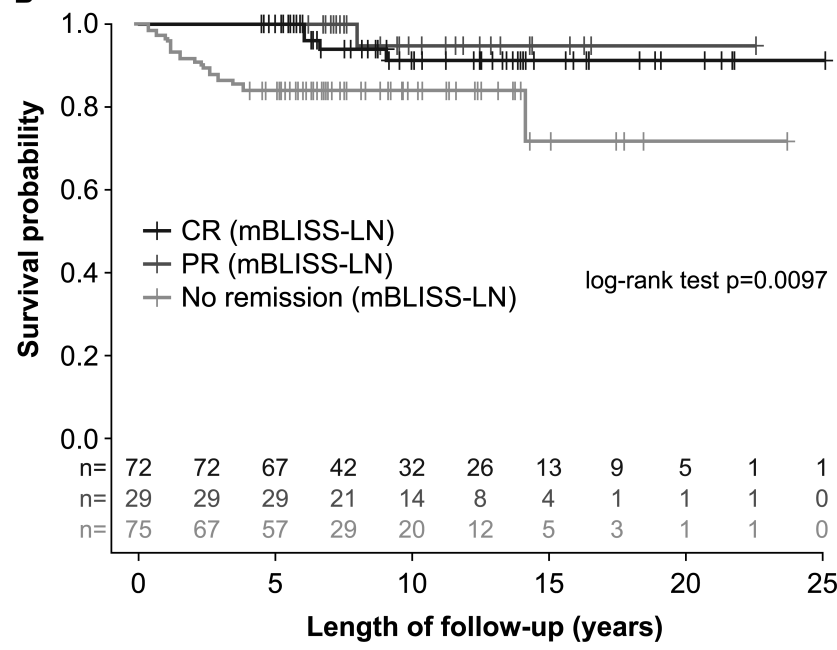

C

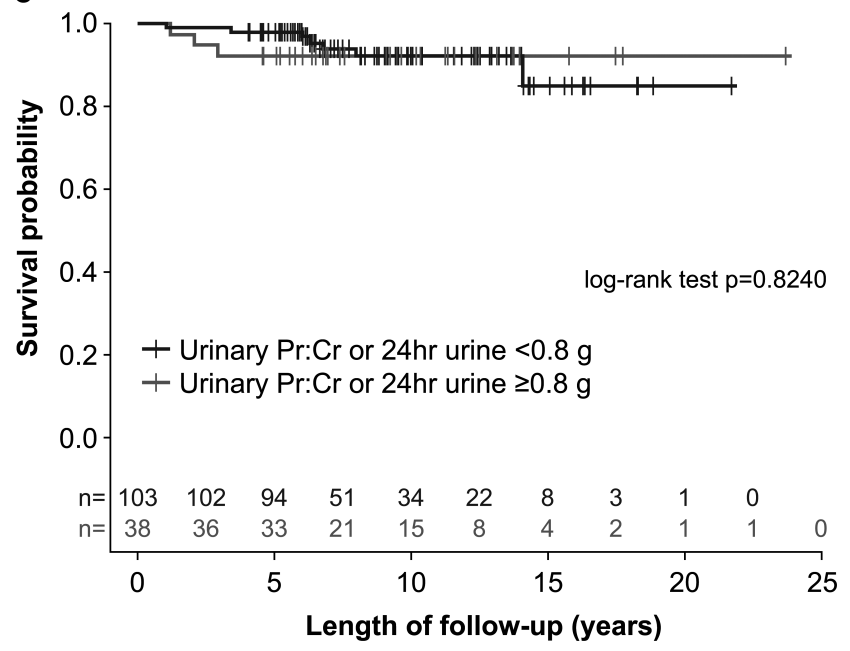

Figure 2. Longterm renal survival based on remission category at 24 months after biopsy. Cutoff of $0.8 \mathrm{~g} /$ day urinary $\mathrm{Pr}: \mathrm{Cr}$ or 24 -h urine selected because of a 2015 publication ${ }^{16}$. A. mALMS criteria (ordinal). B. mBLISS-LN criteria (ordinal). C. Proteinuria (binary criteria). LN: lupus nephritis; CR: complete remission; mALMS: modified Aspreva Lupus Management Study; mBLISS-LN: modified Belimumab International Lupus Nephritis Study; PR: partial remission; Pr:Cr: protein:creatinine.

\section{Personal non-commercial use only. The Journal of Rheumatology Copyright @ 2018. All rights reserved.}




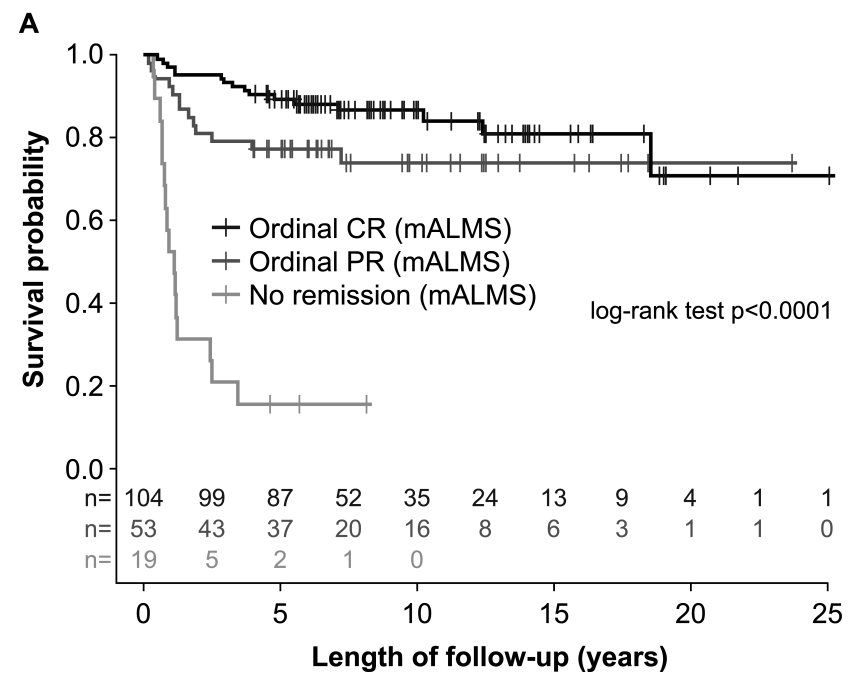

B

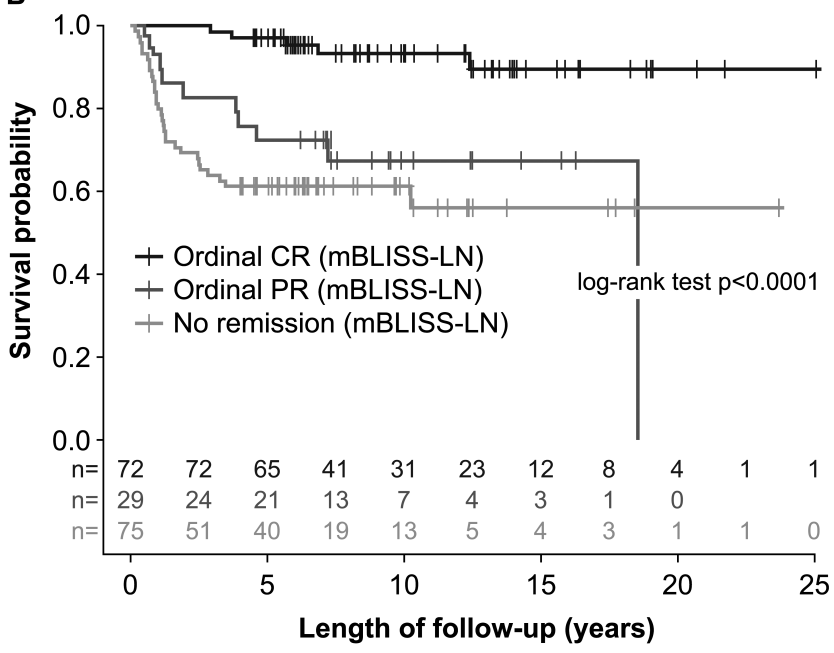

C

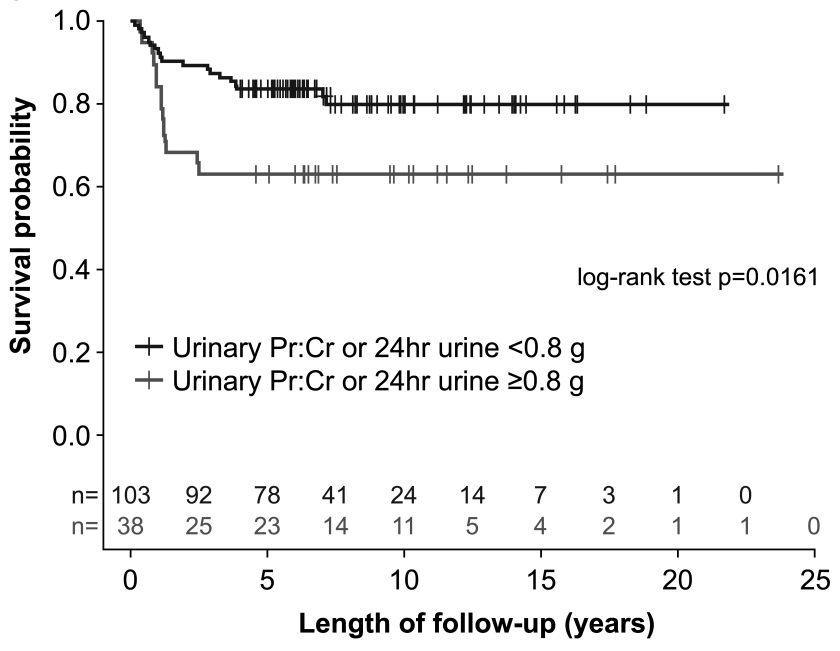

Figure 3. Longterm chronic renal insufficiency-free survival based on status at 24 months. Cutoff of $0.8 \mathrm{~g} /$ day urinary $\mathrm{Pr}: \mathrm{Cr}$ or 24-h urine selected because of a 2015 publication ${ }^{16}$. A. mALMS criteria (ordinal). B. mBLISS-LN criteria (ordinal). C. Proteinuria (binary criteria). LN: lupus nephritis; CR: complete remission; mALMS: modified Aspreva Lupus Management Study; mBLISS-LN: modified Belimumab International Lupus Nephritis Study; PR: partial remission; Pr:Cr: protein:creatinine. category. A relatively small percentage of patients with a PR at Month 24 (mALMS criteria, 26.4\%; mBLISS-LN criteria, 20.7\%) achieved CR at Month 36, highlighting that patients with PR may benefit from careful monitoring.

Mean serum creatinine remained stable at normal or close to normal levels between years 1 to 3 for those in CR or PR (for both the ordinal mALMS and mBLISS-LN criteria); serum creatinine levels as measured at 24 months tended to persist over the following year. In patients with no remission at 24 months (for both the ordinal mALMS and mBLISS-LN criteria), serum creatinine levels remained elevated yet decreased slightly between years 1 and 3, suggesting a possible improvement over time.

The Hopkins Lupus Cohort is a prospective, longitudinal study of disease activity, organ damage, and quality of life in patients with $\mathrm{SLE}^{20}$. The large LN cohort size (> 500 patients) together with the extensive recording of key outcomes provides a rich source of data for investigating the clinical importance of achieving renal remission according to various definitions. There are several limitations to our study, however. The Hopkins Lupus Cohort is a single-center study, which may limit the generality of these results. The sample size meant that the power to detect small differences across groups (e.g., no remission vs PR) was limited. Retrospectively applying clinical trial endpoint criteria to these real-world data may have been associated with some misclassification. Finally, renal response was assessed at 24 months after LN biopsy date in the current study. Future studies could examine the association between renal response measured at earlier (e.g., at 6, 12, and 18 mos) and later timepoints (e.g., at 36 mos) to inform the selection of the optimal timepoint to assess remission status.

Further observational studies in a larger multicenter population are required to confirm these findings and to further assess the clinical relevance of PR.

\section{ACKNOWLEDGMENT}

Medical writing assistance was provided by Louisa Pettinger, $\mathrm{PhD}$, and Jennie McLean, PhD, of Fishawack Indicia Ltd, and was funded by GlaxoSmithKline.

\section{ONLINE SUPPLEMENT}

Supplementary material accompanies the online version of this article.

\section{REFERENCES}

1. Cervera R, Khamashta MA, Font J, Sebastiani GD, Gil A, Lavilla P, et al. Morbidity and mortality in systemic lupus erythematosus during a 10-year period: a comparison of early and late manifestations in a cohort of 1,000 patients. Medicine 2003; 82:299-308.

2. Bernatsky S, Boivin JF, Joseph L, Manzi S, Ginzler E, Gladman DD, et al. Mortality in systemic lupus erythematosus. Arthritis Rheum 2006;54:2550-7.

3. Sprangers B, Monahan M, Appel GB. Diagnosis and treatment of lupus nephritis flares - an update. Nat Rev Nephrol 2012;8:709-17.

4. Maroz N, Segal MS. Lupus nephritis and end-stage kidney disease. Am J Med Sci 2013;346:319-23. 
5. Yap DY, Tang CS, Ma MK, Lam MF, Chan TM. Survival analysis and causes of mortality in patients with lupus nephritis. Nephrol Dial Transplant 2012;27:3248-54.

6. Inda-Filho A, Neugarten J, Putterman C, Broder A. Improving outcomes in patients with lupus and end-stage renal disease. Semin Dial 2013;26:590-6.

7. Furie R, Nicholls K, Cheng TT, Houssiau F, Burgos-Vargas R, Chen SL, et al. Efficacy and safety of abatacept in lupus nephritis: a twelve-month, randomized, double-blind study. Arthritis Rheumatol 2014;66:379-89.

8. Rovin BH, Furie R, Latinis K, Looney RJ, Fervenza FC, Sanchez-Guerrero J, et al. Efficacy and safety of rituximab in patients with active proliferative lupus nephritis: The lupus nephritis assessment with rituximab study. Arthritis Rheum 2012;64:1215-26.

9. Sinclair A, Appel G, Dooley MA, Ginzler E, Isenberg D, Jayne D, et al. Mycophenolate mofetil as induction and maintenance therapy for lupus nephritis: Rationale and protocol for the randomized, controlled Aspreva Lupus Management Study (ALMS). Lupus 2007;16:972-80

10. Appel GB, Contreras G, Dooley MA, Ginzler EM, Isenberg D, Jayne D, et al. Mycophenolate mofetil versus cyclophosphamide for induction treatment of lupus nephritis. J Am Soc Nephrol 2009;20:1103-12.

11. Houssiau FA, Vasconcelos C, D’Cruz D, Sebastiani GD, Garrido Ed Ede R, Danieli MG, et al. Immunosuppressive therapy in lupus nephritis: the Euro-Lupus Nephritis Trial, a randomized trial of low-dose versus high-dose intravenous cyclophosphamide. Arthritis Rheum 2002;46:2121-31.
12. Wofsy D, Hillson JL, Diamond B. Comparison of alternative primary outcome measures for use in lupus nephritis clinical trials. Arthritis Rheum 2013;65:1586-91.

13. Chen YE, Korbet SM, Katz RS, Schwartz MM, Lewis EJ. Value of a complete or partial remission in severe lupus nephritis. Clin J Am Soc Nephrol 2008;3:46-53

14. Hochberg MC. Updating the American College of Rheumatology revised criteria for the classification of systemic lupus erythematosus. Arthritis Rheum 1997;40:1725.

15. Petri M, Orbai AM, Alarcon GS, Gordon C, Merrill JT, Fortin PR, et al. Derivation and validation of the Systemic Lupus International Collaborating Clinics classification criteria for systemic lupus erythematosus. Arthritis Rheum 2012;64:2677-86.

16. Dall'Era M, Cisternas MG, Smilek DE, Straub L, Houssiau FA, Cervera R, et al. Predictors of long-term renal outcome in lupus nephritis trials: lessons learned from the Euro-Lupus Nephritis cohort. Arthritis Rheumatol 2015;67:1305-13.

17. Bose B, Silverman ED, Bargman JM. Ten common mistakes in the management of lupus nephritis. Am J Kidney Dis 2014;63:667-76.

18. Cockcroft DW, Gault MH. Prediction of creatinine clearance from serum creatinine. Nephron 1976;16:31-41.

19. Gladman D, Ginzler E, Goldsmith C, Fortin P, Liang M, Urowitz M, et al. The development and initial validation of the Systemic Lupus International Collaborating Clinics/American College of Rheumatology damage index for systemic lupus erythematosus. Arthritis Rheum 1996;39:363-9.

20. Fangtham M, Petri M. 2013 update: Hopkins lupus cohort. Curr Rheumatol Rep 2013;15:360. 